\title{
Functional RIG-I-like receptors control the survival of mesenchymal stem cells
}

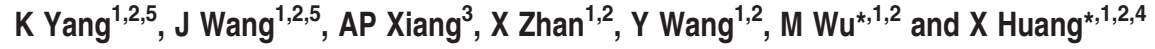

Because of their potent regenerative and immunomodulatory properties, mesenchymal stem cells (MSCs) have promising therapeutic benefits in clinical treatment of inflammatory and infectious diseases. Recent studies suggest that many biological activities of MSCs are largely determined by pattern recognition receptors (PRRs), such as Toll-like receptors (TLRs). However, the role of PRRs in regulating the survival of MSCs remains unknown. In the present study, we examined the viability of MSCs after stimulation of distinct PRRs. Activation of TLRs by direct addition with their respective ligands showed no significant effect on the survival of MSCs, whereas transfection with double-stranded RNA (dsRNA) resulted in marked cell death in MSCs. Transfection of dsRNA upregulated cytosolic retinoic acid inducible gene I (RIG-I)-like receptors (RLRs), including RIG-I and melanoma differentiation-associated antigen 5 (MDA5). Moreover, transfection of dsRNA activated downstream transcription factors interferon regulatory factor 3 (IRF3) and nuclear factor $\kappa \mathrm{B}(\mathrm{NF}-\kappa \mathrm{B})$, as well as induced the expression of interferon- $\beta$ (IFN- $\beta$ ) and pro-inflammatory cytokine interleukin 6 (IL-6) via RLR signaling. Furthermore, we found that transfection of dsRNA triggered both extrinsic and intrinsic apoptotic responses via RLRs. However, ectopic expression of RIG-I or MDA5 was not sufficient to induce apoptosis of MSCs without dsRNA transfection. Our study also revealed that $1 \kappa B$ kinase $\alpha / \beta(I K K \alpha / \beta)$ was required for RLR-mediated apoptosis in MSCs, while TANK-binding kinase 1 (TBK1)/IKK $\varepsilon$ served a pro-survival role. Moreover, neither overexpression of B-cell lymphoma 2 (Bc/2) nor neutralizing autocrined IFN- $\beta$ reduced RLR-mediated apoptosis. In addition, autophagy was induced upon activation of RLRs, however, blocking autophagy did not rescue MSCs from the dsRNA-induced cell death. To the best of our knowledge, this is the first study to explore the role of RLRs in controlling the survival of MSCs, which may provide a clue to understand the pathogenesis of viral infection in MSCs.

Cell Death and Disease (2013) 4, e967; doi:10.1038/cddis.2013.504; published online 12 December 2013

Subject Category: Immunity

Mesenchymal stem cells (MSCs) are a heterogeneous subset of stromal stem cells that can be readily isolated from many adult tissues. They can differentiate into different cell types of the mesodermal lineage, such as adipocytes, osteoblasts and chondrocytes. ${ }^{1}$ In addition to their multipotentiality, MSCs can interact with various immune cells, including T lymphocytes, ${ }^{2}$ macrophages, ${ }^{3}$ and modulate both innate and adaptive immune responses. ${ }^{4}$ These unique properties prompt increasing clinical applications of MSCs in the field of regenerative medicine ${ }^{5}$ and in the treatment of autoimmune and infectious diseases. ${ }^{6}$

Recent studies demonstrate that biological functions of MSCs are largely dependent on distinct pattern recognition receptors (PRRs) ${ }^{7-9}$ which can be activated by various pathogen-associated molecular patterns (PAMPs), such as bacterial lipopolysaccharide (LPS), and viral doublestranded RNA (dsRNA). For example, LPS activates Toll-like receptor (TLR) 4 and increases osteogenic differentiation of MSCs, ${ }^{8}$ whereas dsRNA activates TLR3 and promotes MSC migration, ${ }^{10}$ and enhances the immunomodulatory activity of MSCs in sustaining the neutrophil function. ${ }^{11}$ To date, TLRs (TLR2, TLR3 and TLR4) ${ }^{7,8}$ and nucleotide-binding and oligomerization domain (NOD)-like receptors (NLRs) (NOD1 and NOD2) ${ }^{9}$ have been identified as functional PRRs in MSCs. Nonetheless, nothing is known regarding the expression and function of retinoic

\footnotetext{
${ }^{1}$ Department of Immunology, Zhongshan School of Medicine, Institute of Human Virology, Sun Yat-sen University, Guangzhou 510080, China; ${ }^{2}$ Key Laboratory of Tropical Diseases Control (Sun Yat-sen University), Ministry of Education, Guangzhou 510080, China; ${ }^{3}$ Center for Stem Cell Biology and Tissue Engineering, Sun Yat-sen University, Guangzhou 510080, China and ${ }^{4}$ State Key Laboratory of Ophthalmology, Zhongshan Ophthalmic Center, Sun Yat-sen University, Guangzhou 510060, China

*Corresponding authors: M Wu or X Huang, Department of Immunology, Zhongshan School of Medicine, Sun Yat-sen University, 74 Zhongshan 2nd Road, Guangzhou 510080, China. Tel: +86 020 87334049; Fax: +86 020 87334049; E-mail: wuminhao@ @mail.sysu.edu.cn (MW) or Tel: +86 02087335818 ; Fax: +86 020 87335818; E-mail: huangxi6@mail.sysu.edu.cn $(\mathrm{XH})$

${ }^{5}$ The authors contributed equally to this work.

Keywords: mesenchymal stem cells; RIG-I-like receptors; cell survival; apoptosis; autophagy

Abbreviations: MSCs, mesenchymal stem cells; PRRs, pattern recognition receptors; TLR, Toll-like receptor; PAMPs, pathogen-associated molecular patterns; LPS, lipopolysaccharide; dsRNA, double-stranded RNA; NLR, nucleotide binding and oligomerization domain (NOD)-like receptor; RIG-I, retinoic acid inducible gene I; RLR, RIG-I-like receptor; MDA5, melanoma differentiation-associated antigen 5; IFN, interferon; IPS-1, interferon (IFN)- $\beta$ promoter stimulator 1; TBK1, TANK-binding kinase 1; IKK, I $\kappa$ B kinase; IRF3, interferon regulatory factor 3; NF- $\kappa$ B, nuclear factor $\kappa B$; ISGs, interferon-stimulated genes; PKR, RNA-activated protein kinase; Bcl2, B-cell lymphoma 2; poly(l:C), polyinosinic polycytidylic acid; siRNA, small interference RNA; DAPI, 4,6-diamidino-2-phenylindole; PI, propidium iodide; PARP, poly (ADP-ribose) polymerase; HIV-1, human immunodeficiency virus-1

Received 14.8.13; revised 11.11.13; accepted 13.11.13; Edited by G Melino
} 
acid inducible gene I (RIG-I)-like receptors (RLRs) in MSCs.

As a common viral PAMP, dsRNA has been demonstrated to modulate several biological functions of MSCs. ${ }^{8,11,12}$ This non-self-RNA can be recognized by distinct PRRs and initiates innate antiviral responses. ${ }^{13}$ Whereas extracellular dsRNA accesses to endosome by endocytosis and activates TLR3 on the endosomal membrane, ${ }^{14,15}$ cytosolic dsRNA is recognized by RLRs in the cytosol. ${ }^{16}$

RIG-I and melanoma differentiation-associated antigen 5 (MDA5) are well-characterized RLR family members and selectively recognize short and long dsRNA, respectively. ${ }^{17}$ Upon recognition of dsRNA, RLRs associate with a common adaptor protein, interferon (IFN)- $\beta$ promoter stimulator 1 (IPS-1), which subsequently activates TANK-binding kinase 1 (TBK1), I $\kappa$ B kinase epsilon $\left(\mathrm{IKK} \varepsilon\right.$ ) and IKK $\alpha / \beta .^{18}$ The RLR signaling finally results in the activation of transcription factors interferon regulatory factor 3 (IRF3) and nuclear factor $\kappa \mathrm{B}$ (NF- $\kappa \mathrm{B}$ ) for the induction of type I IFNs and pro-inflammatory cytokines such as interleukin 6 (IL-6). Type I IFNs secreted by viral-infected cells induce the expression of many antiviral interferon-stimulated genes (ISGs), which can restrict virus replication by degrading viral RNA, inhibiting protein synthesis or inducing cell apoptosis. Therefore, the synthetic dsRNA analog polyinosinic polycytidylic acid (poly $(\mathrm{I}: \mathrm{C})$ ) is widely used as an adjuvant in prophylaxis and treatment of viral infection. ${ }^{19,20}$ However, the role of PRRs and PAMPs in the survival of MSCs is largely unknown.

During viral infection or dsRNA stimulation, RLRs have been demonstrated to participate in apoptosis. ${ }^{21-23}$ RLR signaling can directly induce several pro-apoptotic molecules, such as phorbol-12-myristate-13-acetate-induced protein 1 (PMAIP1, also known as NOXA), ${ }^{21,24}$ a BH3-only protein that initiates an intrinsic apoptotic signaling. RLR-induced type I IFNs can initiate or facilitate a pro-apoptotic signaling by inducing some ISGs, including RNA-activated protein kinase (PKR). ${ }^{25,26}$ In addition, activation of RLRs induces the expression of tumor necrosis factor-related apoptosis-inducing ligand (TRAIL), resulting in the initiation of the extrinsic apoptotic pathway in an autocrine manner. ${ }^{27,28}$ In addition to apoptosis, recent studies reveal that RLRs also mediate another cellular stress response, autophagy, ${ }^{29}$ which is a ubiquitous cytosolic homeostasis process in all eukaryotes. Autophagy is rapidly activated in response to extracellular stress, such as starvation, hypoxia and infection. ${ }^{30}$ In most cases, autophagy constitutes an essential mechanism of adaptation to external or internal stress to avoid cell death. ${ }^{31}$ However, massive and extensive autophagy may act as a programmed cell death mechanism and result in autophagic cell death. ${ }^{32}$

In this study, we tested the effect of various PAMPs on the survival of MSCs. Our data demonstrate that activation of TLRs 1-9 has little effect on the survival of MSCs, whereas activation of RIG-I and MDA5 by transfected dsRNA results in marked cell death. Transfected dsRNA induces both extrinsic and intrinsic apoptotic responses via RLR signaling, independently of autocrined IFN- $\beta$. In addition, potent autophagy is triggered in MSCs upon RLR activation but is not responsible for RLR-mediated cell death. Our findings first explore the existence of functional RLRs in MSCs and demonstrate their potential role in controlling the MSC survival, which may provide a better understanding of MSC response to viral infection.

\section{Results}

Transfection of poly(I:C) induces cell death of MSCs. To test whether PRRs have effect on the survival of MSCs, cells were stimulated with indicated PAMPs, and cell viability was examined by using an MTT assay. Activation of TLR2/6, TLR1/2, TLR4, TLR5, TLR7/8 or TLR9 did not reduce the viability of MSCs (Figure 1a), whereas activation of TLR3 by adding poly(l:C) with a high molecular weight (poly $(\mathrm{l}: \mathrm{C})$ $\mathrm{HMW}$ ) or low molecular weight (poly(I:C)-LMW) into the culture media slightly decreased the MSC viability at a concentration of $25 \mu \mathrm{g} / \mathrm{ml}$ (Figure 1a). We further detected the cell viability by using Annexin V/PI staining and flow cytometry analysis. Consistently, stimulation with TLR ligands did not result in cell death of MSCs, except for activation of TLR3 by poly(I:C)-HMW or -LMW led to $5 \%$ cell death of MSCs (Figure 1b). Futhermore, we delivered poly(I:C) into cytosol of MSCs by lipofectin transfection. Both poly(I:C)-HMW and -LMW transfection markedly decreased the viability of MSCs in a dose-dependent manner (Figure 1c). Flow cytometry analysis associated with Annexin $\mathrm{V} / \mathrm{PI}$ staining showed that more than $35 \%$ of MSCs died in the presence of $1 \mu \mathrm{g} / \mathrm{ml}$ poly $(\mathrm{l}: \mathrm{C})$ at $12 \mathrm{~h}$ post transfection (Figure 1d). Morphologically, poly(l:C) transfection induced the adherent cells to round up and detach from the culture dish (Figure 1e). These results indicate that transfection of poly(l:C) induces cell death of MSCs.

\section{Transfection of poly(I:C) upregulates RIG-I and MDA5 in} MSCs. As transfection of poly(l:C) activates cytosolic RLRs, we further detected the expression of RIG-I and MDA5 after poly(l:C) transfection. Real-time PCR data showed that both poly(I:C)-HMW and -LMW transfection upregulated the mRNA expression of RIG-I in a time-dependent manner, peaked at $12 \mathrm{~h}$ post transfection (Figure 2a). The mRNA expression level of MDA5 in poly(I:C)-transfected MSCs was $\sim 35$-fold higher at $4 \mathrm{~h}$ post transfection, when compared with unstimulated MSCs (Figure 2b). However, no change in expression of RIG-I (Figure 2c) or MDA5 (Figure 2d) was detected after addition of poly(I:C)-HMW or -LMW at $100 \mathrm{ng} / \mathrm{ml}$ to the culture media of MSCs. Consistent with mRNA expression, both RIG-I and MDA5 were robustly upregulated at protein level after transfection of poly $(\mathrm{I}: \mathrm{C})$ (Figure 2e) but not addition of poly(l:C) (Figure 2f), as determined by western blot.

RLR stimulation induces activation of IRF3 and NF- $\kappa$ B in MSCs. To investigate the functionality of RLRs in MSCs, we stimulated MSCs by transfection of poly(I:C) to activate RLR signaling and examined the phosphorylation of IRF3 and nuclear translocation of NF- $\kappa \mathrm{B}$. Western blot data showed that both poly(I:C)-HMW and -LMW transfection led to the phosphorylation of IRF3 in a time-dependent manner (Figure 3a). However, direct addition of poly(I:C) at $100 \mathrm{ng} / \mathrm{ml}$ to the culture media of MSCs failed to induce phosphorylation of IRF3 (Figure 3b). Furthermore, nuclear 


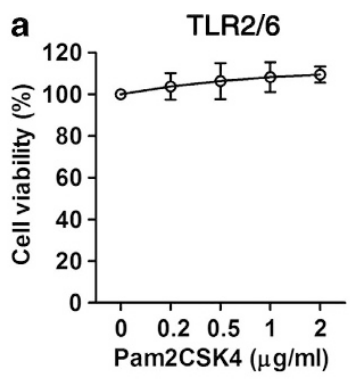

TLR4

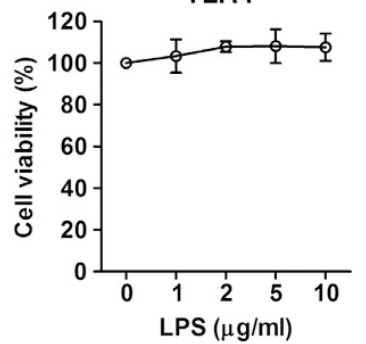

b
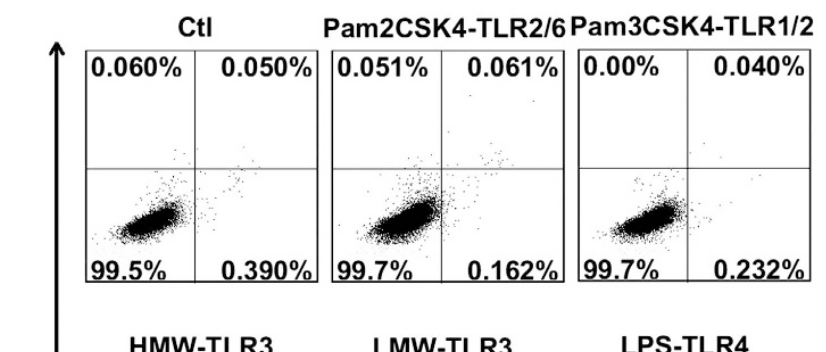

LMW-TLR3
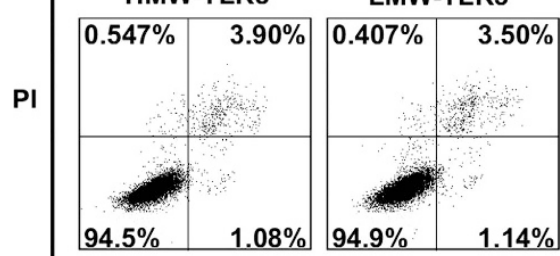

LPS-TLR4

Flagellin-TLR5

R848-TLR7/8
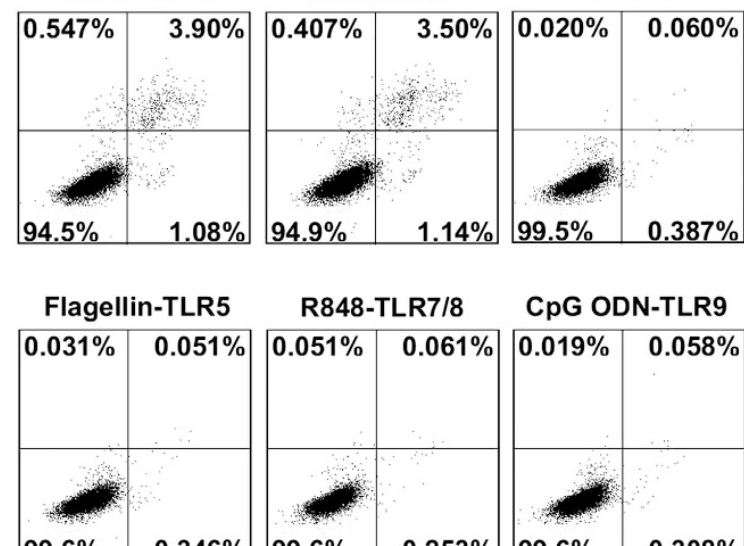

$99.6 \%$

TLR5
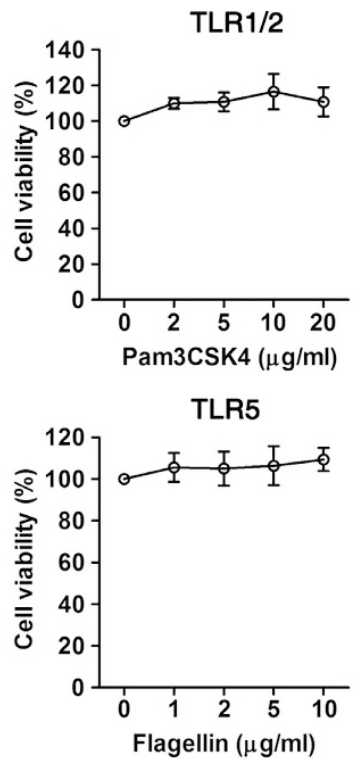

c
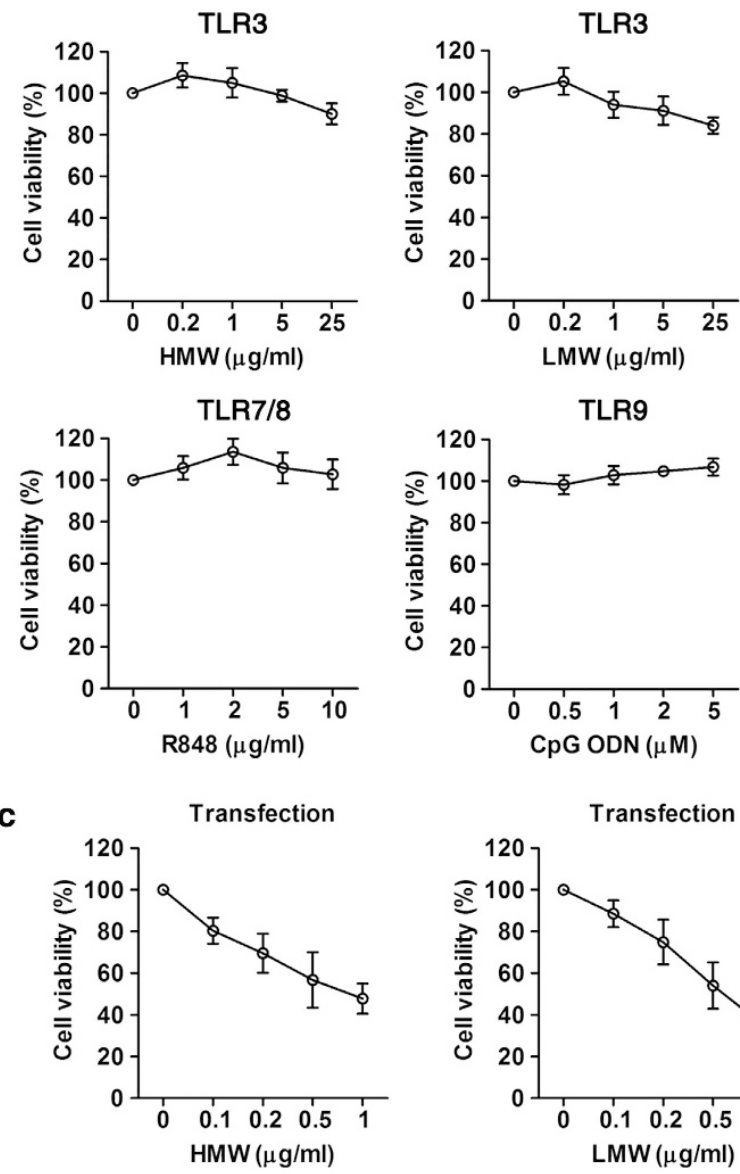

TLR9
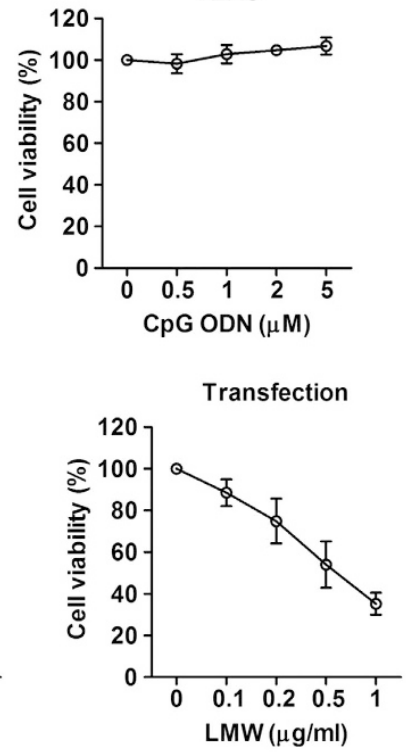

d
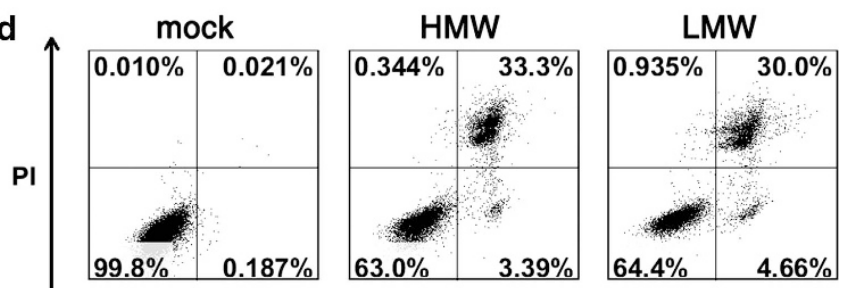

Annexin V-FITC

e
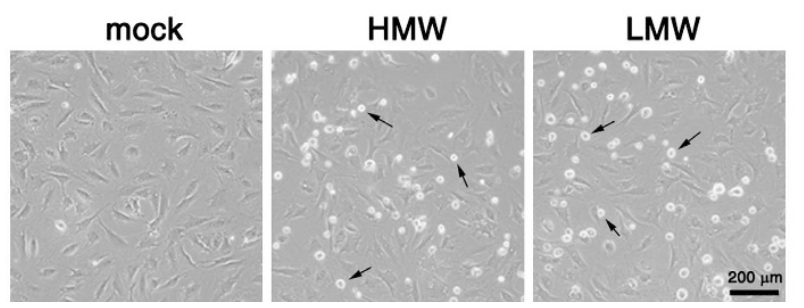

Figure 1 Effect of PRR activation on the survival of MSCs. Individual PAMP was added into the culture media of MSCs at indicated concentrations, and the cell viability was measured by using an MTT assay (a) and flow cytometry analysis with Annexin V/PI staining (b). Poly(l:C)-HMW or -LMW was transfected into MSCs, and the cell viability was measured by using an MTT assay (c) and flow cytometry analysis with Annexin V/PI staining (d). MTT assay data are shown as mean \pm S.E.M. of three independent experiments. Flow cytometric data are representative of three independent experiments with similar results. MSCs were transfected with poly $(\mathrm{l}: \mathrm{C})-\mathrm{HMW}$ or $-\mathrm{LMW}(0.5 \mu \mathrm{g} / \mathrm{ml})$ and examined under a microscope and photographed at $24 \mathrm{~h}$ post treatment (e). Arrows denote rounding and detaching dead cells

translocation of $\mathrm{NF}-\kappa \mathrm{B}$ p65 subunit in poly(I:C)-HMW and -LMW versus mock-transfected MSCs was visualized by immunofluorescence microscopy. In mock-treated MSCs, positive staining for $\mathrm{NF}-\kappa \mathrm{B}$ p65 subunit was mainly distributed in the cytoplasm, whereas, in poly(I:C)-HMW and -LMW-transfected MSCs, NF- $\kappa$ B p65 protein aggregated in the nuclei of MSCs (Figure 3c). In addition, in MSCs directly stimulated with poly(I:C), no nuclear translocation of 

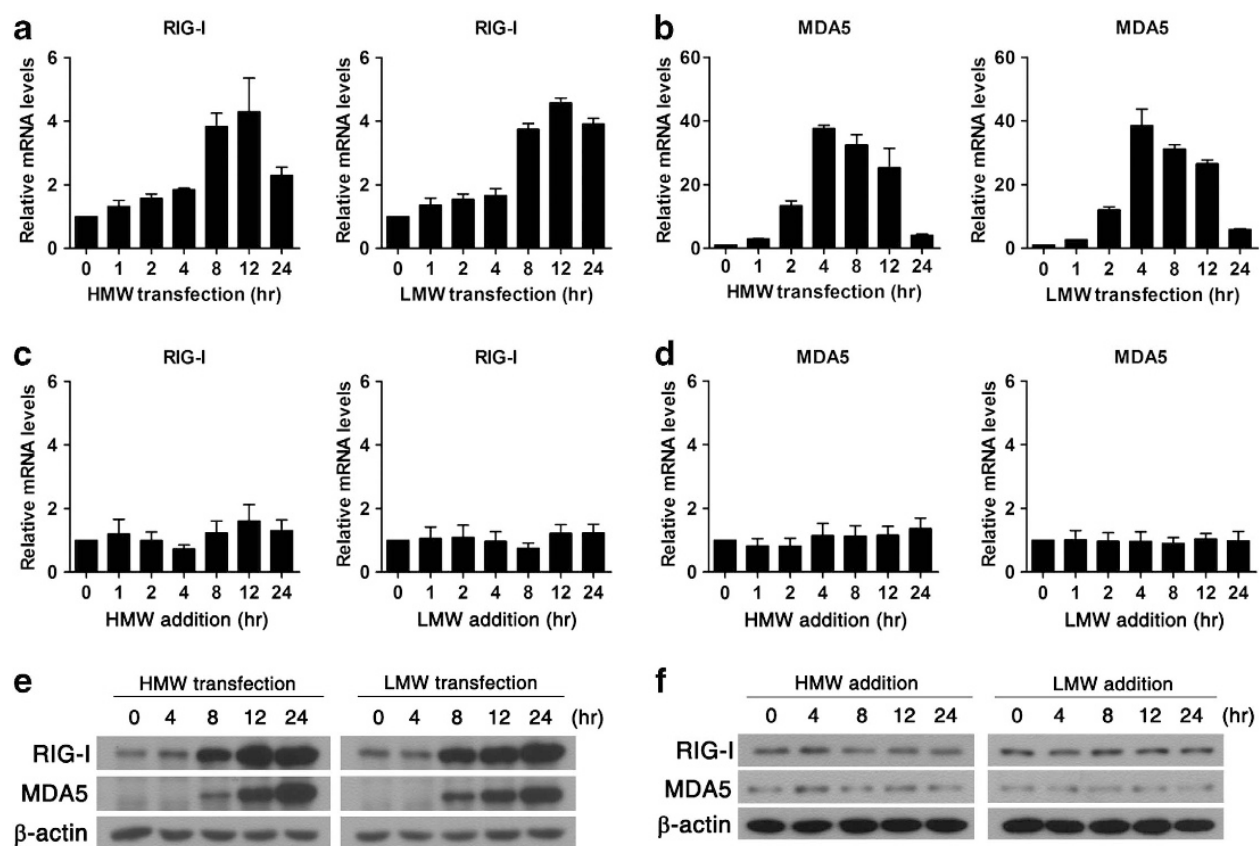

Figure 2 Transfection of poly(I:C) upregulates RIG-I and MDA5 in MSCs. MSCs were transfected or added with poly(l:C)-HMW or -LMW at $100 \mathrm{ng} / \mathrm{ml}$ for the indicated time. mRNA expression levels of RIG-I (a and $\mathbf{c}$ ) and MDA5 ( $\mathbf{b}$ and $\mathbf{d}$ ) were analyzed by real-time PCR. Data are shown as mean \pm S.E.M. of three independent experiments. The protein expression levels of RIG-I and MDA5 were analyzed by western blot in MSCs after poly(l:C) transfection (e) or addition (f). Data are representative of three independent experiments with similar results
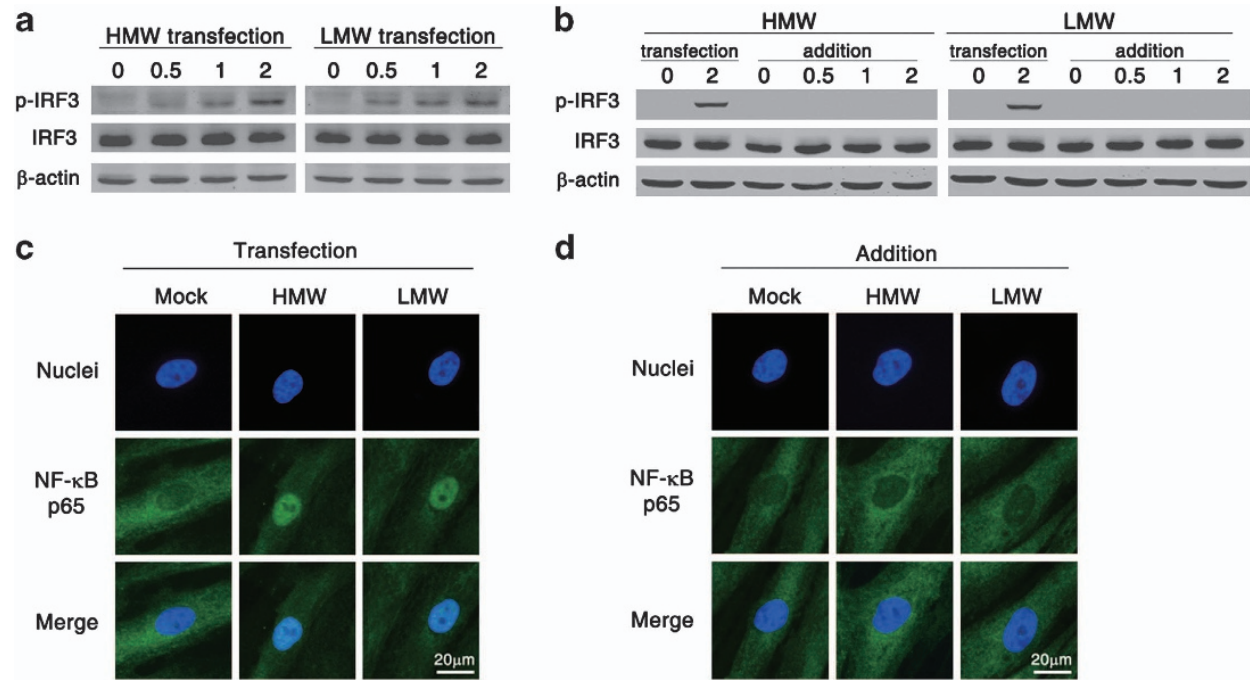

d

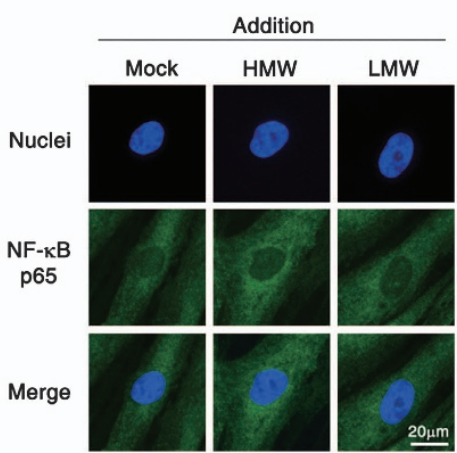

Figure 3 Activation of RLRs induces activation of IRF3 and NF- $\kappa$ B in MSCs. Poly(l:C)-HMW or -LMW (100 ng/ml) was transfected into MSCs (a) or added to the culture media (b) for the indicated time, and the phosphorylation of IRF3 was detected by western blot. The nuclear translocation of NF- $\kappa$ B p65 subunit (green) was monitored by immunofluorescence microscopy after poly(l:C) transfection (c) or addition (d). Nuclei were visualized by DAPI staining (blue). Data are representative of three independent experiments with similar results

the NF- $\kappa$ B p65 subunit was observed (Figure 3d). Collectively, these data indicate that RLRs stimulation in MSCs results in the activation of downstream IRF3 and NF- $\kappa$ B signaling pathways.

Activation of RLRs triggers the expression of IFN- $\beta$ and IL-6 in MSCs. We further used real-time PCR to detect the expression of IFN- $\beta$ and pro-inflammatory cytokine IL-6, which are downstream effector genes of RLR signaling.
IFN- $\beta$ was induced in a dose-dependent manner in MSCs transfected with either poly(l:C)-HMW or -LMW (Figure 4a). IFN- $\beta$ expression increased rapidly after poly(I:C) transfection as early as $1 \mathrm{~h}$ post treatment, peaking at $8 \mathrm{~h}$ post treatment (Figure 4b). However, stimulation of MSCs by addition of poly $(\mathrm{I}: \mathrm{C})$ to culture media did not induce IFN- $\beta$ expression (Figures $4 \mathrm{c}$ and d). ELISA data further confirmed that transfection of poly $(\mathrm{I}: \mathrm{C})$ induced the production of IFN- $\beta$ in a time-dependent manner (Figure $4 \mathrm{e}$ ). In addition, mRNA 
a

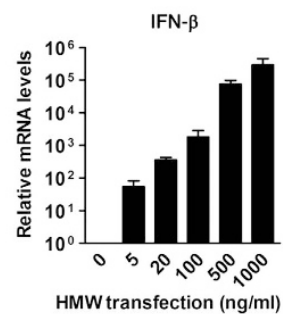

C

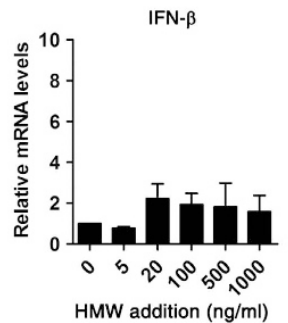

e

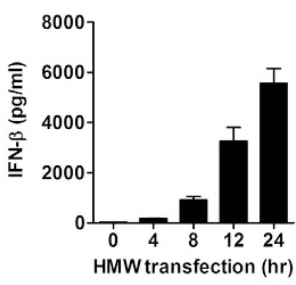

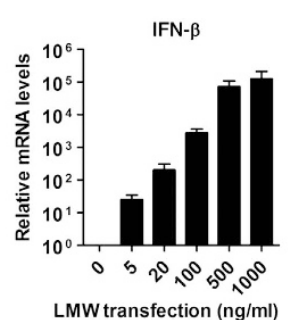

IFN- $\beta$

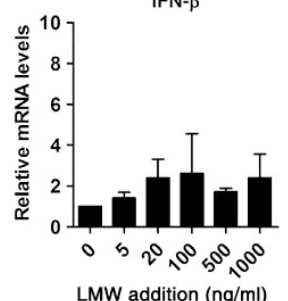

LMW addition (ng/ml)
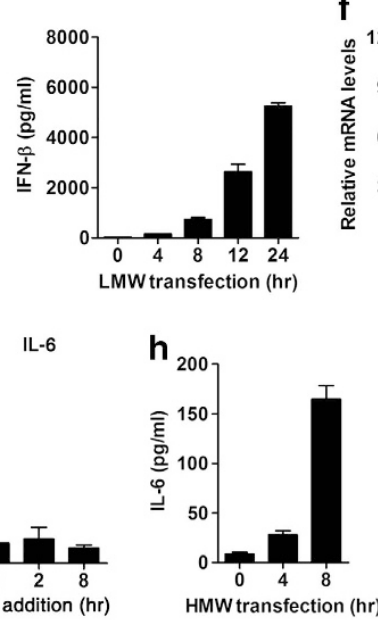

HMW transfection (hr) b

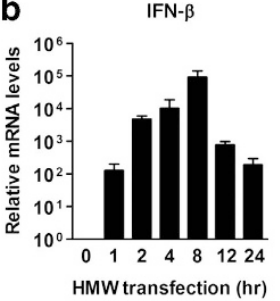

d
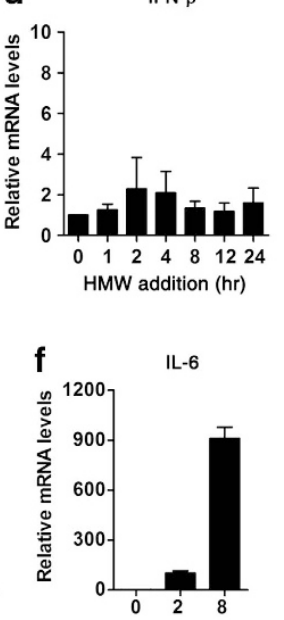

HMW transfection (hr)

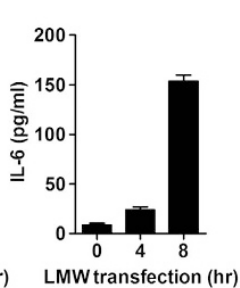

IFN- $\beta$

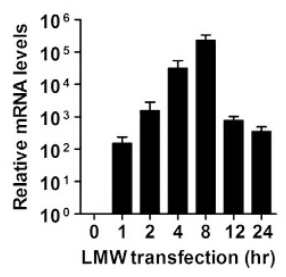

IFN- $\beta$

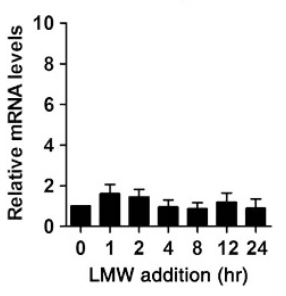

IL-6

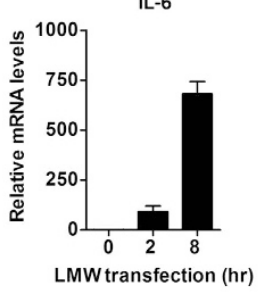

i

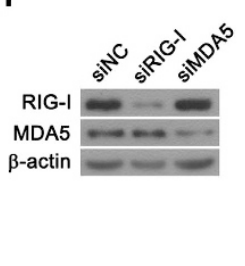

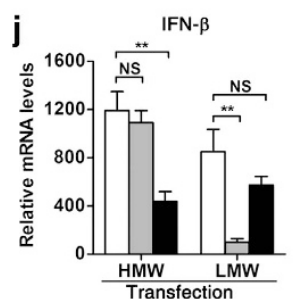

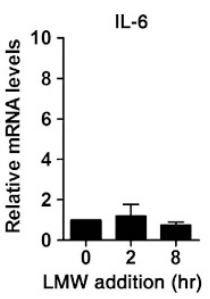

IL-6
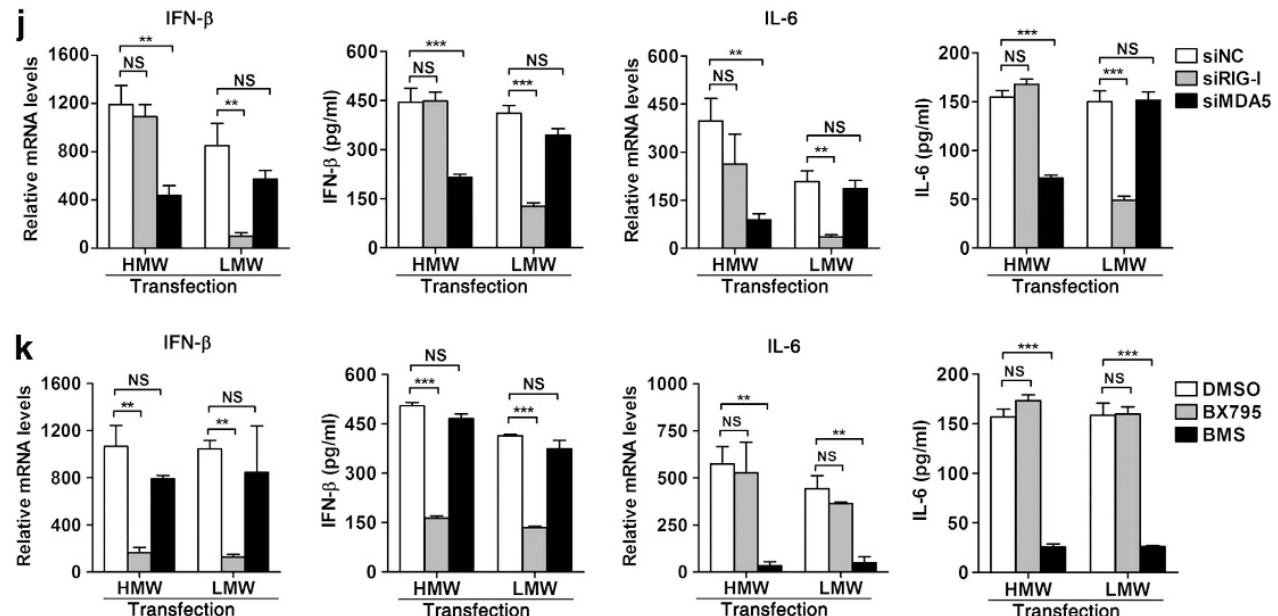

Figure 4 Activation of RLRs induces the expression of IFN- $\beta$ and IL-6. Poly(I:C)-HMW or -LMW was transfected into MSCs or added to the culture media for the indicated concentration or time. mRNA levels of IFN- $\beta$ (a-d) and IL-6 (f and $\mathbf{g}$ ) were analyzed by real-time PCR. Protein levels of IFN- $\beta$ (e) and IL-6 (h) in supernatants of poly(l:C)transfected MSCs were measured by ELISA. MSCs were transfected with siRNA targeting RIG-I and MDA5 for $48 \mathrm{~h}$ (i and $\mathbf{j})$, or pretreated with inhibitors for TBK1//KK $\varepsilon$ $(\mathrm{BX} 795,1 \mu \mathrm{M})$ or IKK $\alpha / \beta(\mathrm{BMS}-345541,10 \mu \mathrm{M})$ for $1 \mathrm{~h}(\mathbf{k})$, followed by poly(I:C) transfection. The knockdown efficacy was confirmed by western blot (i). mRNA and protein levels of IFN- $\beta$ and IL- 6 were detected by real-time PCR and ELISA, respectively (j and $\mathbf{k}$ ). Data are shown as mean \pm S.E.M. of three independent experiments. ${ }^{* \star} P<0.01$; ${ }^{* * *} P<0.001 ;$ NS, not significant 
expression of pro-inflammatory cytokine IL-6 was increased after poly $(\mathrm{I}: \mathrm{C})$ transfection (Figure $4 \mathrm{f}$ ) but not addition to culture media (Figure 4g). ELISA data showed that production of IL-6 was enhanced in MSCs after poly(I:C) transfection (Figure 4h). To further confirm the functionality of RLRs in MSCs, RNA interference technique was used to knockdown endogenous expression of RIG-I and MDA5. The silencing efficiency of siRIG-I and siMDA5 was confirmed by western blot (Figure 4i). Both real-time PCR and ELISA data indicated that silencing of RIG-I decreased IFN- $\beta$ and IL-6 induction in poly(I:C)-LMW-transfected cells, whereas silencing of MDA5 had no significant effects on LMW-induced IFN- $\beta$ or IL-6 production (Figure $4 \mathrm{j}$ ). In contrast, both IFN- $\beta$ and IL- 6 induction by HMW transfection was reduced by siMDA5 but not siRIG-I (Figure 4j). These results suggest that transfection of poly(I:C)-LMW and -HMW in MSCs activates RIG-I and MDA5, respectively. Together, these data indicate that both RIG-I and MDA5 are functionally expressed in MSCs. We further tested the involvement of $\mathrm{TBK} 1 / \mathrm{IKK} \varepsilon / \mathrm{IRF} 3$ and $\mathrm{IKK} \alpha / \beta / \mathrm{NF}-\kappa \mathrm{B}$ signaling pathways in RLR-mediated cytokine production. Inhibition of TBK1/IKK $\varepsilon$ with BX795 attenuated IFN- $\beta$ production induced by poly(I:C) transfection but had little effect on the induction of IL-6 (Figure 4k). In contrast, the production of IL-6 in poly(I:C)transfected MSCs was significantly reduced after blocking $\mathrm{NF}-\kappa \mathrm{B}$ signaling with an IKK $\alpha / \beta$ inhibitor BMS-345541, whereas no difference was detected in IFN- $\beta$ production (Figure 4k). These results suggest that TBK1/IKK $/$ /IRF3 signaling mediated the induction of IFN- $\beta$ in poly(I:C)transfected MSCs, whereas IKK $\alpha / \beta / \mathrm{NF}-\kappa \mathrm{B}$ signaling mediated the induction of IL-6.

Activation of RLRs induces apoptosis in MSCs. To determine whether apoptosis is involved in poly(I:C)-induced cell death of MSCs, we detected the cleavage of Caspase-3 and its substrate poly (ADP-ribose) polymerase (PARP), which are specific hallmarks of apoptotic cell death. Western blot data showed that poly(l:C) transfection led to the cleavage of Caspase-3 and PARP in MSCs in a time- and dose-dependent manner (Figures $5 \mathrm{a}$ and b), indicating the activation of apoptosis. However, direct addition of poly(I:C) to culture media at a concentration as high as $25 \mu \mathrm{g} / \mathrm{ml}$ induced weak apoptosis of MSCs, suggesting that efficient apoptosis induction of dsRNA in MSCs required cytosolic delivery (Figure 5c). Furthermore, in LMW-treated MSCs, knockdown of RIG-I significantly inhibited cell apoptosis, whereas HMW-induced apoptosis was reduced by silencing of MDA5 (Figure 5d), suggesting that RLRs mediated pro-apoptotic signaling induced by cytosolic dsRNA. Interestingly, ectopic expression of RIG-I or MDA5 without poly(I:C) transfection did not induce the cleavage of Caspase-3 or PARP (Figure 5e). However, overexpression of RIG-I in MSCs promoted LMW-induced apoptosis, whereas overexpression of MDA5 enhanced HMW-induced apoptosis (Figure 5e). We also found that inhibition of TBK1/IKK $\varepsilon$ increased poly(I:C)-induced apoptosis of MSCs, whereas $\mathrm{IKK} \alpha / \beta$ inhibitor rescued MSCs from apoptosis (Figure 5f). These results indicate that $\mathrm{IKK} \alpha / \beta$ mediates a pro-apoptotic signaling, whereas $\mathrm{TBK} 1 / \mathrm{IKK} \varepsilon$ serves a prosurvival role. We also tested the cleavage of Caspase- 8 and
Caspase-9, which are initiator caspases of extrinsic and intrinsic apoptotic pathways, respectively. Transfection of HMW or LMW into MSCs resulted in the cleavage of both Caspase-8 and Caspase-9 (Figure $5 \mathrm{~g}$ ), indicating RLR stimulation activated both extrinsic and intrinsic apoptotic pathways. Furthermore, overexpression of Bcl2 (Figure 5h) or blockage of IFN- $\beta$ with a neutralizing antibody (Figure 5i) had little effect on poly(l:C)-induced apoptosis of MSCs, suggesting that the poly(l:C)-induced apoptosis is independent of $\mathrm{Bcl} 2$ and IFN- $\beta$.

Activation of RLRs induces autophagy but not autophagic cell death in MSCs. In addition to apoptosis, activation of RLRs by transfection of dsRNA induces autophagy, another cellular stress response that may result in autophagic cell death ultimately. Western blot data showed that transfection with either poly(I:C)-HMW or -LMW resulted in a conversion from LC3 I to LC3 II (Figure 6a), indicating the induction of autophagy in MSCs. Moreover, the autophagic response in MSCs was monitored by monodansylcadaverine (MDC) staining, a specific autophagosome indicator. Accumulation of MDC-positive autophagosome was observed in poly(I:C)-transfected MSCs (Figure 6b). In addition, immunofluorescence microscopy showed that stimulation of MSCs with poly $(\mathrm{l}: \mathrm{C})$ led to redistribution of LC3 from a diffuse to punctate pattern, which is characteristic of autophagosomes (Figure 6c). Altogether, these data above indicate that autophagy is induced in MSCs upon RLR activation.

Furthemore, to determine whether autophagy mediates dsRNA-induced cell death of MSCs, 3-methyladenine (3-MA), LY294002 (LY) or Wortmannin (WM) was used to block the autophagic response in MSCs. Western blot data showed that all three inhibitors were able to block dsRNA-induced autophagy (Figure 6d). However, none of them had significant impact on dsRNA-induced MSCs cell death (Figure 6e). Similarly, silencing of Beclin1, a key autophagy-related gene, markedly decreased the expression levels of LC3 II but not cleaved Caspase-3 or PARP, suggesting that knockdown of Beclin1 led to the blockage of autophagic but not apoptotic response in MSCs (Figure 6f). In addition, knockdown of Beclin1 failed to rescue MSCs from dsRNA-induced cell death (Figure $6 \mathrm{~g}$ ), indicating that, although autophagy is robustly induced in MSCs upon activation of RLR signaling, it is not responsible for RLRs-mediated cell death of MSCs.

\section{Discussion}

MSCs acquire great interests recently for their potential application in clinical therapy of injury and immune-related disorders due to their regenerative ${ }^{5}$ and immunosuppressive properties. ${ }^{33}$ The biological activities of MSCs, including differentiation, ${ }^{7,8}$ migration and immunomodulation, ${ }^{10}$ are largely determined by various functional PRRs that recognize either extracellular or cytosolic signals.

RLRs are cytosolic RNA helicases responsible for recognizing viral PAMPs such as dsRNA. ${ }^{16}$ In the present study, we identify the existence of functional RLRs in MSCs for the first time. Both RIG-I and MDA5 are constitutively expressed in MSCs and upregulated after dsRNA 
a

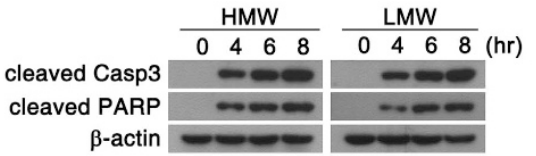

C

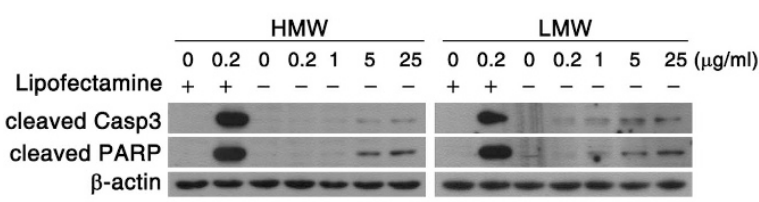

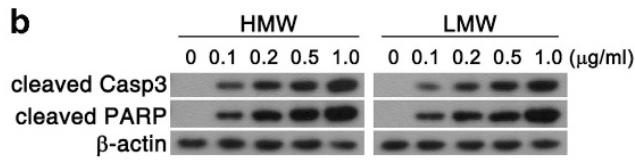

d

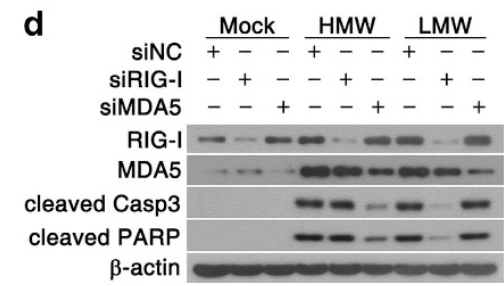

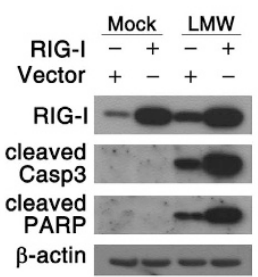

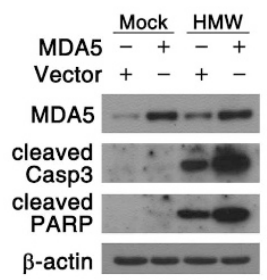

f

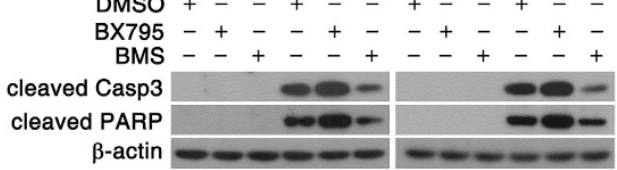

h

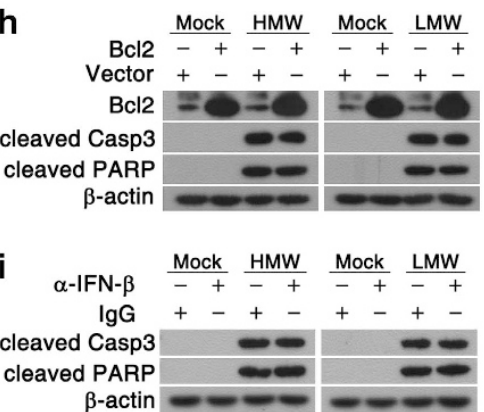

Figure 5 Activation of RLRs induces apoptosis in MSCs. Apoptosis signaling-related molecules in MSCs were tested by western blot. MSCs were transfected with poly(l:C) (HMW or LMW, $200 \mathrm{ng} / \mathrm{ml})$ for the indicated time (a) or for $6 \mathrm{~h}$ at the indicated concentration (b). MSCs were stimulated with poly(l:C) complexed with or without Lipofectemine 2000 for $6 \mathrm{~h}$ (c). MSCs were transfected with siRNA targeting RIG-I and MDA5 (d) or expressing plasmid of RIG-I, MDA5 (e) or Bcl2 (h), followed by poly(l:C) transfection $(200 \mathrm{ng} / \mathrm{ml})$ for $6 \mathrm{~h}$. MSCs were pretreated with inhibitors for TBK1/IKK $\varepsilon(B X 795,1 \mu \mathrm{M})$ or IKK $\alpha / \beta(\mathrm{BMS}-345541,10 \mu \mathrm{M})(\mathrm{f})$, or incubated with $500 \mathrm{U} / \mathrm{ml}$ anti-IFN- $\beta$ neutralizing antibody $(\alpha-\mathrm{IFN}-\beta)(\mathrm{i})$, followed by poly(l:C) transfection $(200 \mathrm{ng} / \mathrm{ml})$ for $6 \mathrm{~h}$. MSCs were transfected with poly(l:C) at the indicated concentration for $6 \mathrm{~h}(\mathrm{~g})$. Data are representative of three independent experiments with similar results

transfection. Activation of RIG-I and MDA5 by dsRNA transfection induces phosphorylation of IRF3, nuclear translocation of NF- $\kappa \mathrm{B}$ and expression of IFN- $\beta$ and IL-6, indicating that RLRs expressed in MSCs are functional.

Previous studies have identified other functional PRRs in MSCs, including TLR2, TLR3 and TLR4, which have a critical role in modulating proliferation, differentiation, ${ }^{7,8}$ migration and immunoregulatory activity. ${ }^{10}$ However, whether PRRs influence the MSC survival remains unknown. It is reported that TLRs differentially regulate cell death of several other cell types. For example, activation of TLR2 triggers microglial cell death $^{34}$ but does not lead to cell death in B lymphocytes. ${ }^{35}$ TLR4 activation reduces the survival of intestinal stem cells ${ }^{36}$ but promotes cancer cell survival in hepatocellular carcinoma. ${ }^{37}$ These reports suggest that TLRs induce cell death in a context-dependent manner. Our study shows that activation of RLRs markedly reduces the viability of MSCs, whereas activation of TLRs has no influence on MSC survival, except for TLR3 activation induces a slight decrease in cell viability, indicating that RLR signaling is critical in controlling MSC survival. To the best of our knowledge, this is the first study to explore the role of PRRs in regulating the survival of MSCs.

Apoptosis is a common type of programmed cell death that largely influences cell viability. It is reported that serum deprivation and hypoxia can induce the apoptotic response in MSCs. ${ }^{38}$ However, which PRR participates in modulating MSC apoptosis is still unclear. Our study demonstrates that transfection of dsRNA results in the cleavage of apoptotic marker Caspase-3 and PARP in MSCs, whereas addition of dsRNA only slightly induces apoptotic response till reaching a concentration as high as $25 \mu \mathrm{g} / \mathrm{ml}$. This observation is consistent with other study showing the marked difference in caspase activation and apoptoic response in extracellular versus cytosolic dsRNA-stimulated human macrophages or keratinocytes. $^{39}$ It is well known that dsRNA with different length could be recognized by distinct RNA sensors. Experimentally, transfection of short or long dsRNA activates the cytosolic receptor RIG-I and MDA5, respectively, ${ }^{17}$ whereas addition of both types of dsRNA activated TLR3 expressed on the endosomal membrane. ${ }^{14,15}$ Studies have demonstrated that RLR activation induces rapid apoptosis in 
a

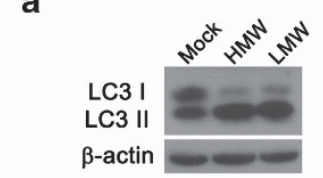

b
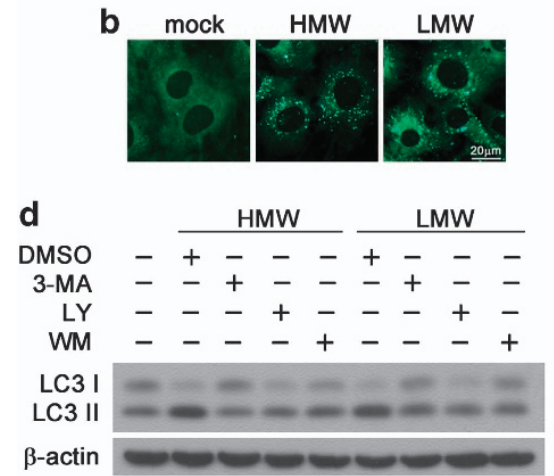

f

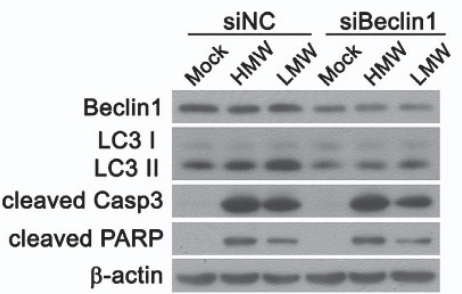

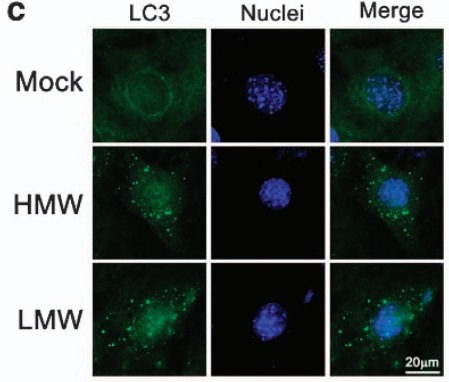

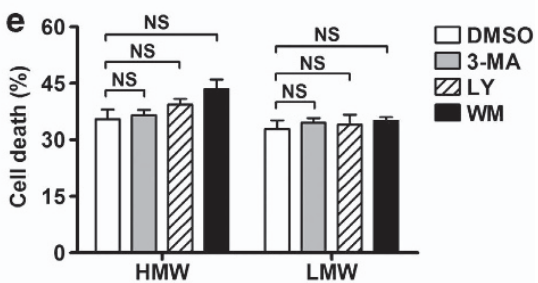

g

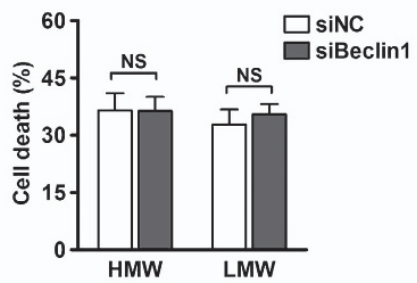

Figure 6 RLR stimulation activates autophagy but not autophagic cell death in MSCs. MSCs were transfected with poly(l:C) (HMW or LMW, $200 \mathrm{ng} / \mathrm{ml})$ or mock treated for $6 \mathrm{~h}$. (a) Protein levels of LC3 I and LC3 II were analyzed by western blot. (b) Autophagic vacuoles were stained by using monodansylcadaverine (MDC) for 30 min and visualized with fluorescence microscopy. (c) The distribution of endogenous LC3 was detected with immunofluorescence microscopy. MSCs were pretreated with 3-MA (10 mM), LY294002 (LY, $10 \mu \mathrm{M})$ or Wortmannin (WM, $200 \mathrm{nM}$ ) for $1 \mathrm{~h}$ before poly(l:C) transfection (HMW or LMW, $200 \mathrm{ng} / \mathrm{ml})$. (d) Protein levels of LC3 I and LC3 II were detected with western blot at $6 \mathrm{~h}$ after transfection. (e) Cell death was measured by using an MTT assay at $24 \mathrm{~h}$ after transfection. MSCs were transfected with siBeclin1 or siNC for $48 \mathrm{~h}$, followed by poly(l:C) transfection (HMW or LMW, $200 \mathrm{ng} / \mathrm{ml})$. (f) The conversion of LC3 and cleavage of Caspase-3 and PARP were detected by western blot at $6 \mathrm{~h}$ after poly(l:C) transfection. (g) Cell death was measured by using an MTT assay at $24 \mathrm{~h}$ after poly $(\mathrm{l}: \mathrm{C})$ transfection. MTT data are shown as mean \pm S.E.M. of three independent experiments. NS, not significant

various cancer cell types, such as human melanoma cells. ${ }^{22}$ In the present study, knockdown of endogenous RLRs attenuates the dsRNA-induced apoptosis in MSCs, further confirming that the pro-apoptotic effect of cytosolic dsRNA is RLR-dependent. Previous studies showed that knockout of RIG-I or MDA5 impaired the production of IFN- $\beta$ and antiviral defense after infection with RNA viruses. ${ }^{40}$ Ectopic expression of RIG-I did not result in the induction of IFN- $\beta$ but enhanced the induction of IFN- $\beta$ after either dsRNA transfection or RNA virus infection. ${ }^{41}$ Similarly, in our study, ectopic expression of RIG-I or MDA5 is not sufficient to induce apoptosis of MSCs but increases apoptosis induced by dsRNA transfection.

The IKK-related kinases, TBK1 and IKK $\varepsilon$, are wellcharacterized regulators of the innate immune response because of their abilities to phosphorylate transcription factor IRF3 ${ }^{42}$ Recent evidence showed that both TBK1 and IKK 8 contribute to cell survival. ${ }^{43}$ TBK1 suppresses apoptosis in oncogenic KRAS-transformed cells, ${ }^{44}$ while IKK $\varepsilon$ is required for the survival of MCF-7 breast cancer cells. ${ }^{45}$ Our results show that inhibition of TBK1//KK $\varepsilon$ significantly increased the dsRNA-induced MSC apoptosis, suggesting the critical pro-survival role of $\mathrm{TBK} 1 / \mathrm{IKK} \varepsilon$ in MSCs. Studies have demonstrated that $\mathrm{NF}-\kappa \mathrm{B}$ functions to promote resistance to cell death through induction of antiapoptotic genes ${ }^{46}$ but may also promote apoptosis under certain circumstances. ${ }^{47,48}$ In this study, we found that poly(l:C)-induced apoptosis of MSCs is reduced upon suppression of IKK $\alpha / \beta$, suggesting the pro-apoptotic role of NF- $\kappa$ B signaling in MSCs.

Studies have revealed that RLR signaling triggers either extrinsic or intrinsic apoptotic pathways. For example, in human melanoma cells, RLR signaling induced the proapoptotic BH3-only proteins Puma and Noxa independently of type I IFNs and resulted in efficient activation of intrinsic apoptosis. ${ }^{22}$ Whereas in Sendai virus-infected prostate cancer cells ${ }^{28}$ or dsRNA-transfected breast cancer cells, ${ }^{49}$ both extrinsic and intrinsic apoptotic signaling pathways are initiated by RLR activation. In our study, activation of RLR signaling triggers cleavage of both Caspase-8 and Caspase-9, indicating RLRs mediate both extrinsic and intrinsic apoptosis. However, overexpression of Bcl2, a key antiapoptotic molecule regulator of the intrinsic apoptotic pathway, does not reduce apoptosis in poly(l:C)-transfected MSCs, indicating that the intrinsic pathway has a minor role in the poly(I:C)-induced apoptosis in MSCs. In addition, activation of RLRs leads to robust production of IFN- $\beta$, which may be involved in modulating cell apoptosis. For instance, modified Vaccinia virus Ankara infection activated cytosolic RLRs to 
initiate apoptosis in an IFN- $\beta$-dependent manner. ${ }^{21}$ However, in our study, blocking IFN- $\beta$ with a eutralizing antibody do not rescue MSCs from RLR-mediated apoptosis, suggesting the autocrined IFN- $\beta$ do not mediate cytosolic dsRNA-induced apoptosis of MSCs. This finding is consistent with other studies showing that activation of RLRs induces apoptotic response independently of type I IFN in human melanoma cells $^{22}$ or in the absence of type I IFN receptor. ${ }^{50}$

In addition to apoptosis, autophagy is another cellular stress response that is related to cell survival. It is reported that dsRNA transfection induces apoptosis of breast cancer cells, which is accompanied by autophagy. ${ }^{49}$ Our study demonstrates that activation of RLR signaling by transfected dsRNA triggers both apoptotic and autophagic responses. Autophagy is an evolutionarily conserved self-digestion process and constitutes a stress adaptation that protects cells against death. However, it may also serve as an alternative cell death pathway, designated as autophagic cell death. Tormo et al. report that in melanoma cells, transfected dsRNA induces autophagy via RLR signaling, which in turn leads to dramatic autophagic cell death. ${ }^{29}$ Whereas Inao et al. demonstrates that autophagy plays a cytoprotective role in human breast cancer cells by suppressing the dsRNAinduced apoptosis. ${ }^{49}$ In the present study, we find that, although autophagy is induced after dsRNA transfection, it does not influence the dsRNA-induced cell death or apoptosis in MSCs. On the basis of literature, induction of autophagy is beneficial for clearance of some intracellular pathogens such as viruses; ${ }^{51}$ thus, we hypothesize that the cytosolic dsRNAtriggered autophagic response may have an important role in the antiviral response by MSCs, which needs further investigation.

Several clinical investigations reveal the susceptibility of MSCs to a variety of viruses, such as human immunodeficiency virus-1 (HIV-1) ${ }^{52}$ It is reported that purified genomic RNA from HIV is sensed by RIG-I, ${ }^{53}$ which may explain the observation of HIV-1-induced apoptosis in MSCs. ${ }^{52}$ Therefore, our work has important implication for understanding the pathogenesis of viral infection in MSCs.

In summary, our study first explores the expression and function of RLRs in MSCs. We demonstrate that transfected dsRNA activates cytosolic RLR signaling and induces expression of IFN- $\beta$ and pro-inflammatory cytokine IL-6. Moreover, activation of RLR signaling by cytosolic dsRNA triggers marked apoptotic response independently of INF- $\beta$. In addition, autophagic response is triggered in MSCs upon RLR activation but not responsible for RLR-mediated cell death. Altogether, our data provide a better understanding of RLRs in regulating the survival of MSCs, which may gain further insights into the pathogenesis of viral infection in MSCs.

\section{Materials and Methods}

Reagents. Synthetic bacterial lipoprotein Pam2CSK4, Pam3CSK4, synthetic dsRNA mimic poly(l:C)-HMW or -LMW, LPS (from E. coli 0111:B4), ultrapure flagellin (from S. typhimurium), R848, synthetic CpG oligonucleotide (ODN 1668) and TBK1//KK sinhibitor (BX795) were purchased from Invivogen (San Diego, CA, USA). IKK $\alpha / \beta$ inhibitor (BMS-345541), LY294002, Wortmannin were from Merck Millipore (Darmstadt, Germany). Monodansylcadaverine (MDC), 3-methyladenine, anti- $\beta$-actin antibody and rabbit IgG were from Sigma (St. Louis, MO, USA). Anti-RIG-I, anti-MDA5, anti-cleaved Caspase-3, anti-cleaved PARP, anti-cleaved
Caspase-8, anti-cleaved Caspase-9 and anti-phosphorylated IRF3 antibodies were obtained from Cell Signaling Technology (Beverly, MA, USA). Anti-NF- $\kappa$ B p65 and anti-IRF3 antibodies were obtained from Santa Cruz Biotechnology (Santa Cruz, CA, USA). Anti-LC3 antibody was from Novus Biologicals (Littleton, CO, USA). Anti-Beclin1 antibody was from R\&D Systems (Minneapolis, MN, USA). Anti-IFN- $\beta$ neutralizing antibody was from PBL interferon source (Piscataway, NJ, USA). Anti-Bcl2 antibody was from R\&D systems (Minneapolis, MN, USA).

Cell culture. MSCs were obtained from bone marrow of tibia and femur of 6- to 8-week-old C57BL/6 mice as described previously. ${ }^{54}$ Cells were cultured in DMEM low-glucose medium supplemented with $10 \%$ FBS, $2 \mathrm{mM}$ glutamine, $100 \mathrm{U} / \mathrm{ml}$ penicillin and $100 \mathrm{mg} / \mathrm{ml}$ streptomycin (all from Invitrogen, Carlsbad, CA, USA). The isolated MSCs were immunophenotyped by flow cytometry as reported previously. ${ }^{54}$ MSCs from Passage 5 to 20 were used in all experiments. All experiments involving animals were approved by the Animal Ethics Committee of Sun Yat-Sen University.

Cell stimulation. To stimulate TLRs $1-9$, their respective ligands were added directly into the culture media. To stimulate RIG-I and MDA5, poly(I:C)-HMW or -LMW was delivered into cytoplasm of MSCs by using Lipofectamine 2000 (Invitrogen) according to the manufacturer's protocol. For mock transfection, no synthetic poly(l:C) was used in the procedure.

siRNA knockdown. All siRNAs targeting specific genes of interest were obtained from Invitrogen, and scrambled siRNA (siNC) was used as negative control. The target sequences of siRNAs used in the present study were as follows: RIG-I siRNA (siRIG-I), 5'-GCCCAUUGAAACCAAGAAAUU-3'; MDA5 siRNA (siMDA5), 5'-GUCAUUAGUAAAUUUCGCACU-3'; Beclin1 siRNA (siBeclin1), $5^{\prime}$-CAGUUUGGCACAAUCAAUA-3'. For knockdown experiments, cells were transfected with siRNA $(100 \mathrm{nM})$ for $48 \mathrm{~h}$ using Lipofectamine 2000 (Invitrogen) according to the manufacturer's protocol.

Plasmid. To generate $\mathrm{Bcl} 2$ expression vector, full-length mouse $\mathrm{Bc} / 2$ was amplified with PCR and cloned into pcDNA3. pcDNA3-mRIG-I and pcDNA3 were provided by Professor Baoxue Ge (Tongji University, Shanghai, China). pUNO1mcs and pUNO1-mMDA5 were purchased from Invivogen. Transfection of plasmid was performed using the Lipofectamine 2000 reagent (Invitrogen) according to the manufacturer's instructions.

Real-time PCR. Total RNA was isolated with TRIzol reagent (Invitrogen) according to the manufacturer's instruction. First-strand cDNA synthesis was performed using the RevertAid First Strand cDNA Synthesis Kit (Thermo Fisher Scientific, Waltham, MA, USA). The cDNA was quantitated in duplicate using SYBR Green Master Mix (Applied Biosystems, Foster City, CA, USA) as reported. ${ }^{55}$ The specificity of the amplified PCR products was assessed by melting curve analysis. Relative mRNA levels were calculated after normalization to $\beta$-actin. The sequences of primers for RIG-I, MDA5 and IFN- $\beta$ are as following: RIG-I, forward, $5^{\prime}$-GAC GTTCGAGATTCCAGTCAT-3', and reverse, $5^{\prime}$-GCATTGGCTCATCAACTCCAT- $3^{\prime}$; MDA5, forward, 5'-ACCAGATCCCCTGAAGTTGGAGT-3', and reverse, 5'-GGAA GAAGGGTGAACTGAGGAGATG-3'; IFN- $\beta$, forward, $5^{\prime}$-GGAGATGACGGAGAA GATGCAGA-3', and reverse, 5'-GGAGTTCATCCAGGAGACGTACA-3'. Other primers used in the present study were described previously. ${ }^{56,57}$

Enzyme-linked immunosorbent assay. Supernatants from cultured MSCs were collected, and cytokine protein levels were tested using ELISA kits (PBL interferon source Verikine Mouse IFN- $\beta$ ELISA kit and eBioscience Mouse IL-6 Platinum ELISA kit) according to the manufacturer's instructions. The limits of detection of IFN- $\beta$ and IL-6 are 15.6 and $6.5 \mathrm{pg} / \mathrm{ml}$, respectively.

Western blot. Western blot was performed as described previously. ${ }^{58}$ Briefly, $20 \mu \mathrm{g}$ proteins were separated on SDS-PAGE, transferred to polyvinylidene difluoride membrane, blocked with $5 \%(\mathrm{w} / \mathrm{v})$ nonfat milk and then probed with the indicated primary antibody. Western blots were developed using horseradish peroxidase-conjugated secondary antibody, followed by detection with enhanced chemiluminescence.

Immunofluorescence microscopy. Cells were seeded onto coverslips and treated with poly $(\mathrm{I}: \mathrm{C})$ or mock transfection as mentioned above and then fixed with $4 \%$ paraformaldehyde, permeabilized with $0.05 \%$ Triton X-100 and blocked 
with $5 \%$ BSA in PBS. Samples were incubated with a primary antibody at $4{ }^{\circ} \mathrm{C}$ overnight, rinsed three times with PBS and then incubated with Alexa Fluor 488 Goat Anti-Rabbit IgG (Invitrogen) for $1 \mathrm{~h}$ at room temperature. Coverslips were washed twice before 4,6-diamidino-2-phenylindole (DAPI) staining and mounted with ProLong Gold antifade reagent (Invitrogen). Stained samples were visualized using an Olympus BX53 fluorescence microscope (Olympus Corporation, Tokyo, Japan).

Cell viability assay. Cell viability was assessed by using an MTT assay in triplicate. In brief, one-tenth volume of $5 \mathrm{mg} / \mathrm{ml}$ MTT solution was added into cultured media and allowed to incubate for $4 \mathrm{~h}$ at $37^{\circ} \mathrm{C}$ in dark. The violet formazan crystal was dissolved in dimethyl sulfoxide (DMSO) and the absorbance was measured at $490 \mathrm{~nm}$.

Annexin V/propidium iodide staining. MSCs were treated with indicated ligand for $12 \mathrm{~h}$, then collected, resuspended in binding buffer and stained with Annexin V-FITC and propidium iodide (KeyGEN Biotech, Nanjing, China) according to the manufacturer's instructions. The stained cells were analyzed with flow cytometry (Beckman Coulter EPICS XL/MCL, Fullerton, CA, USA). Data were analyzed with Flowjo Software (Tree Star, Ashland, OR, USA).

Statistical analysis. Experiments were performed in duplicate or triplicate for at least three times independently. GraphPad Prism 4.0 (GraphPad Software, San Diego, CA, USA) was used for statistical analysis. The differences between two groups were compared by using an unpaired two-tailed Student's $t$-test, while differences between three groups or more were compared by using analysis of variance (ANOVA) with Bonferroni's post hoc test. Differences were considered statistically significant with a $P$-value less than 0.05 .

\section{Conflict of Interest}

The authors declare no conflict of interest.

Acknowledgements. This work was supported by grants National Natural Science Foundation of China (U0832006, 31200662, 81261160323), Guangdong Innovative Research Team Program (No. 2009010058), Specialized Research Fund for the Doctoral Program of Higher Education of China (20100171110047, 20120171120064), Guangdong Natural Science Foundation (1025100 8901000013, S2012040006680), Guangdong Province Universities and Colleges Pearl River Scholar Funded Scheme (No. 2009), National Science and Technology Key Projects for Major Infectious Diseases (2013ZX10003001), The 111 Project (No. B13037).

1. Pittenger MF, Mackay AM, Beck SC, Jaiswal RK, Douglas R, Mosca JD et al. Multilineage potential of adult human mesenchymal stem cells. Science 1999; 284: 143-147.

2. Meisel R, Zibert A, Laryea M, Gobel U, Daubener W, Dilloo D. Human bone marrow stromal cells inhibit allogeneic T-cell responses by indoleamine 2,3-dioxygenase-mediated tryptophan degradation. Blood 2004; 103: 4619-4621.

3. Nemeth K, Leelahavanichkul A, Yuen PS, Mayer B, Parmelee A, Doi K et al. Bone marrow stromal cells attenuate sepsis via prostaglandin $E(2)$-dependent reprogramming of host macrophages to increase their interleukin-10 production. Nat Med 2009; 15: 42-49.

4. Aggarwal S, Pittenger MF. Human mesenchymal stem cells modulate allogeneic immune cell responses. Blood 2005; 105: 1815-1822.

5. Bernardo ME, Pagliara D, Locatelli F. Mesenchymal stromal cell therapy: a revolution in regenerative medicine? Bone Marrow Transplant 2012; 47: 164-171.

6. Tyndall A, Uccelli A. Multipotent mesenchymal stromal cells for autoimmune diseases: teaching new dogs old tricks. Bone Marrow Transplant 2009; 43: 821-828.

7. Pevsner-Fischer M, Morad V, Cohen-Sfady M, Rousso-Noori L, Zanin-Zhorov A, Cohen S et al. Toll-like receptors and their ligands control mesenchymal stem cell functions. Blood 2007; 109: 1422-1432.

8. Hwa Cho H, Bae YC, Jung JS. Role of toll-like receptors on human adipose-derived stromal cells. Stem Cells 2006; 24: 2744-2752.

9. Kim HS, Shin TH, Yang SR, Seo MS, Kim DJ, Kang SK et al. Implication of NOD1 and NOD2 for the differentiation of multipotent mesenchymal stem cells derived from human umbilical cord blood. PLoS One 2010; 5: e15369.

10. Tomchuck SL, Zwezdaryk KJ, Coffelt SB, Waterman RS, Danka ES, Scandurro AB. Toll-like receptors on human mesenchymal stem cells drive their migration and immunomodulating responses. Stem Cells 2008; 26: 99-107.

11. Cassatella MA, Mosna F, Micheletti A, Lisi V, Tamassia N, Cont C et al. Toll-like receptor-3activated human mesenchymal stromal cells significantly prolong the survival and function of neutrophils. Stem Cells 2011; 29: 1001-1011.
12. Liotta F, Angeli R, Cosmi L, Fili L, Manuelli C, Frosali F et al. Toll-like receptors 3 and 4 are expressed by human bone marrow-derived mesenchymal stem cells and can inhibit their T-cell modulatory activity by impairing Notch signaling. Stem Cells 2008; 26: 279-289.

13. Akira S, Uematsu S, Takeuchi O. Pathogen recognition and innate immunity. Cell 2006; 124: 783-801.

14. Alexopoulou L, Holt AC, Medzhitov R, Flavell RA. Recognition of double-stranded RNA and activation of NF-kappaB by Toll-like receptor 3. Nature 2001; 413: 732-738.

15. Itoh K, Watanabe A, Funami K, Seya T, Matsumoto M. The clathrin-mediated endocytic pathway participates in dsRNA-induced IFN-beta production. J Immunol 2008; 181: 5522-5529.

16. Kato H, Takahasi K, Fujita T. RIG--l-like receptors: cytoplasmic sensors for non-self RNA. Immunol Rev 2011; 243: 91-98.

17. Kato $\mathrm{H}$, Takeuchi $\mathrm{O}$, Mikamo-Satoh $\mathrm{E}$, Hirai $\mathrm{R}$, Kawai $\mathrm{T}$, Matsushita $\mathrm{K}$ et al. Length-dependent recognition of double-stranded ribonucleic acids by retinoic acid-inducible gene-I and melanoma differentiation-associated gene 5. J Exp Med 2008; 205: 1601-1610.

18. Kawai T, Takahashi K, Sato S, Coban C, Kumar H, Kato H et al. IPS-1, an adaptor triggering RIG-I- and Mda5-mediated type I interferon induction. Nat Immunol 2005; 6: 981-988.

19. Zhao J, Wohlford-Lenane C, Fleming E, Lane TE, McCray PB Jr, Perlman S. Intranasal treatment with poly $(1: C)$ protects aged mice from lethal respiratory virus infections. $J$ Virol 2012; 86: 11416-11424.

20. Liang Z, Wu S, Li Y, He L, Wu M, Jiang L et al. Activation of Toll-like receptor 3 impairs the dengue virus serotype 2 replication through induction of IFN-beta in cultured hepatoma cells. PLoS One 2011; 6: e23346.

21. Eitz Ferrer P, Potthoff S, Kirschnek S, Gasteiger G, Kastenmuller W, Ludwig $\mathrm{H}$ et al. Induction of Noxa-mediated apoptosis by modified vaccinia virus Ankara depends on viral recognition by cytosolic helicases, leading to IRF-3/IFN-beta-dependent induction of pro-apoptotic Noxa. PLoS Pathog 2011; 7: e1002083.

22. Besch R, Poeck H, Hohenauer T, Senft D, Hacker G, Berking C et al. Proapoptotic signaling induced by RIG-I and MDA-5 results in type I interferon-independent apoptosis in human melanoma cells. J Clin Invest 2009; 119: 2399-2411.

23. Glas M, Coch C, Trageser D, Dassler J, Simon M, Koch P et al. Targeting the cytosolic innate immune receptors RIG-I and MDA5 effectively counteracts cancer cell heterogeneity in glioblastoma. Stem Cells 2013; 31: 1064-1074.

24. Sun $Y$, Leaman DW. Involvement of Noxa in cellular apoptotic responses to interferon, double-stranded RNA, and virus infection. J Biol Chem 2005; 280: 15561-15568.

25. Zhang P, Samuel CE. Protein kinase PKR plays a stimulus- and virus-dependent role in apoptotic death and virus multiplication in human cells. J Virol 2007; 81: 8192-8200.

26. McAllister CS, Samuel CE. The RNA-activated protein kinase enhances the induction of interferon-beta and apoptosis mediated by cytoplasmic RNA sensors. J Biol Chem 2009; 284: 1644-1651.

27. Harada K, Sato Y, Itatsu K, Isse K, Ikeda H, Yasoshima M et al. Innate immune response to double-stranded RNA in biliary epithelial cells is associated with the pathogenesis of biliary atresia. Hepatology 2007; 46: 1146-1154.

28. Matsushima-Miyagi T, Hatano K, Nomura M, Li-Wen L, Nishikawa T, Saga K et al. TRAIL and Noxa are selectively upregulated in prostate cancer cells downstream of the RIG-I/MAVS signaling pathway by nonreplicating Sendai virus particles. Clin Cancer Res 2012; 18: 6271-6283.

29. Tormo D, Checinska A, Alonso-Curbelo D, Perez-Guijarro E, Canon E, Riveiro-Falkenbach E et al. Targeted activation of innate immunity for therapeutic induction of autophagy and apoptosis in melanoma cells. Cancer Cell 2009; 16: 103-114.

30. Levine B, Kroemer G. Autophagy in the pathogenesis of disease. Cell 2008; 132: $27-42$.

31. Maiuri MC, Zalckvar E, Kimchi A, Kroemer G. Self-eating and self-killing: crosstalk between autophagy and apoptosis. Nat Rev Mol Cell Biol 2007; 8: 741-752.

32. Denton D, Nicolson S, Kumar S. Cell death by autophagy: facts and apparent artefacts. Cell Death Differ 2012; 19: 87-95.

33. Marigo I, Dazzi F. The immunomodulatory properties of mesenchymal stem cells. Semin Immunopathol 2011; 33: 593-602.

34. Arroyo DS, Soria JA, Gaviglio EA, Garcia-Keller C, Cancela LM, Rodriguez-Galan MC et al. Toll-like receptor 2 ligands promote microglial cell death by inducing autophagy. FASEB J 2013; 27: 299-312.

35. Iskra S, Kalla M, Delecluse HJ, Hammerschmidt W, Moosmann A. Toll-like receptor agonists synergistically increase proliferation and activation of $B$ cells by epstein-barr virus. J Virol 2010; 84: 3612-3623.

36. Neal MD, Sodhi CP, Jia H, Dyer M, Egan CE, Yazji I et al. Toll-like receptor 4 is expressed on intestinal stem cells and regulates their proliferation and apoptosis via the p53 up-regulated modulator of apoptosis. J Biol Chem 2012; 287: 37296-37308.

37. Wang L, Zhu R, Huang Z, Li H, Zhu H. Lipopolysaccharide-induced toll-like receptor 4 signaling in cancer cells promotes cell survival and proliferation in hepatocellular carcinoma. Dig Dis Sci 2013; 58: 2223-2236.

38. Zhu W, Chen J, Cong X, Hu S, Chen X. Hypoxia and serum deprivation-induced apoptosis in mesenchymal stem cells. Stem Cells 2006; 24: 416-425.

39. Rintahaka J, Wiik D, Kovanen PE, Alenius H, Matikainen S. Cytosolic antiviral RNA recognition pathway activates caspases 1 and 3 . J Immunol 2008; 180: $1749-1757$. 
40. Kato H, Sato S, Yoneyama M, Yamamoto M, Uematsu S, Matsui K et al. Cell type-specific involvement of RIG-I in antiviral response. Immunity 2005; 23: 19-28.

41. Yoneyama M, Kikuchi M, Natsukawa T, Shinobu N, Imaizumi T, Miyagishi M et al. The RNA helicase RIG-I has an essential function in double-stranded RNA-induced innate antiviral responses. Nat Immunol 2004; 5: 730-737.

42. Fitzgerald KA, McWhirter SM, Faia KL, Rowe DC, Latz E, Golenbock DT et al. IKKepsilon and TBK1 are essential components of the IRF3 signaling pathway. Nat Immunol 2003; 4 491-496.

43. Ou YH, Torres M, Ram R, Formstecher E, Roland C, Cheng T et al. TBK1 directly engages Akt/PKB survival signaling to support oncogenic transformation. Mol Cell 2011; 41: 458-470.

44. Barbie DA, Tamayo P, Boehm JS, Kim SY, Moody SE, Dunn IF et al. Systematic RNA interference reveals that oncogenic KRAS-driven cancers require TBK1. Nature 2009; 462 108-112.

45. Boehm JS, Zhao JJ, Yao J, Kim SY, Firestein R, Dunn IF et al. Integrative genomic approaches identify IKBKE as a breast cancer oncogene. Cell 2007; 129: 1065-1079.

46. Karin M, Lin A. NF-kappaB at the crossroads of life and death. Nat Immunol 2002; 3 221-227.

47. Schneider A, Martin-Villalba A, Weih F, Vogel J, Wirth T, Schwaninger M. NF-kappaB is activated and promotes cell death in focal cerebral ischemia. Nat Med 1999; 5: 554-559.

48. Qin ZH, Chen RW, Wang Y, Nakai M, Chuang DM, Chase TN. Nuclear factor kappaB nuclear translocation upregulates c-Myc and p53 expression during NMDA receptormediated apoptosis in rat striatum. J Neurosci 1999; 19: 4023-4033.

49. Inao T, Harashima N, Monma H, Okano S, Itakura M, Tanaka T et al. Antitumor effects of cytoplasmic delivery of an innate adjuvant receptor ligand, poly(l:C), on human breast cancer. Breast Cancer Res Treat 2012; 134: 89-100.

50. Knowlton JJ, Dermody TS, Holm GH. Apoptosis induced by mammalian reovirus is beta interferon (IFN) independent and enhanced by IFN regulatory factor 3- and NF-kappaBdependent expression of Noxa. J Virol 2012; 86: 1650-1660.
51. Yordy B, Iwasaki A. Autophagy in the control and pathogenesis of viral infection. Curr Opin Virol 2011; 1: 196-203.

52. Gibellini D, Alviano F, Miserocchi A, Tazzari PL, Ricci F, Clo A et al. HIV-1 and recombinant gp120 affect the survival and differentiation of human vessel wall-derived mesenchymal stem cells. Retrovirology 2011; 8: 40.

53. Berg RK, Melchjorsen J, Rintahaka J, Diget E, Soby S, Horan KA et al. Genomic HIV RNA induces innate immune responses through RIG-I-dependent sensing of secondarystructured RNA. PLoS One 2012; 7: e29291.

54. Lei J, Wang Z, Hui D, Yu W, Zhou D, Xia W et al. Ligation of TLR2 and TLR4 on murine bone marrow-derived mesenchymal stem cells triggers differential effects on their immunosuppressive activity. Cell Immunol 2011; 271: 147-156.

55. $\mathrm{Ng} \mathrm{MH}, \mathrm{Ho}$ TH, Kok KH, Siu KL, Li J, Jin DY. MIP-T3 is a negative regulator of innate type I IFN response. J Immunol 2011; 187: 6473-6482.

56. Wu M, Peng A, Sun M, Deng Q, Hazlett LD, Yuan J et al. TREM-1 amplifies corneal inflammation after Pseudomonas aeruginosa infection by modulating Toll-like receptor signaling and Th1/Th2-type immune responses. Infect Immun 2011; 79: 2709-2716.

57. Chen K, Yin L, Nie X, Deng Q, Wu Y, Zhu M et al. beta-Catenin promotes host resistance against Pseudomonas aeruginosa keratitis. J Infect 2013; 67: 584-594.

58. Wu S, He L, Li Y, Wang T, Feng L, Jiang L et al. miR-146a facilitates replication of dengue virus by dampening interferon induction by targeting TRAF6. J Infect 2013; 67: 329-341.

cc) (i) () $\Theta$ Cell Death and Disease is an open-access journal published by Nature Publishing Group. This work is licensed under a Creative Commons Attribution-NonCommercialNoDerivs 3.0 Unported License. To view a copy of this license, visit http://creativecommons.org/licenses/by-nc-nd/3.0/ 